Table 1.

Table 1

\begin{tabular}{|c|c|c|}
\hline Epidemiological and clinical features & Patients (27) & Healthy control (30) \\
\hline Age (mะsd) & $48.7 \pm 11$ & $49.1 \pm 10$ \\
\hline $\mathbf{F} / \mathbf{M}$ & $21 / 6$ & $20 / 10$ \\
\hline \multicolumn{3}{|l|}{ Comorbidity } \\
\hline Hypertension & 4 & 5 \\
\hline Obesity & 2 & 3 \\
\hline Diabetes & 1 & 1 \\
\hline \multicolumn{3}{|l|}{ Rheumatic disease } \\
\hline Psoriatic arthritis/Spondylo-arthritis & $10 / 3$ & \\
\hline Rheumatoid arthritis & 6 & \\
\hline Systemic sclerosis / Sjogren syndrome/SLE & $5 / 1 / 2$ & \\
\hline \multicolumn{3}{|l|}{ Therapy } \\
\hline TNF-i & 5 & \\
\hline Small molecules & 3 & \\
\hline Anti-IL17 & 3 & \\
\hline OH-clorochine & 5 & \\
\hline Methotrexate/Mycophenolate & $4 / 1$ & \\
\hline Anti-il $12 / 23$ & 2 & \\
\hline Anti-IL 6/CTLA4-Ig & $1 / 1$ & \\
\hline Notherapy & 2 & \\
\hline
\end{tabular}

Acknowledgements: I thank the Italian League of Systemic Sclerosis for launching a survey for ssc patients.

Disclosure of Interests: None declared

DOI: 10.1136/annrheumdis-2021-eular.3529

\section{POS1249 MULTISYSTEM INFLAMMATORY SYNDROME IN CHILDREN DURING THE COVID-19 PANDEMIC IN TURKEY: FIRST REPORT FROM THE EASTERN MEDITERRANEAN}

Y. Ozsurekci ${ }^{1}$, S. Gürlevik ${ }^{1}$, S. Kesici ${ }^{2}$, U. Kaya Akca ${ }^{3}$, P. D. Oygar ${ }^{1}$, K. Aykac ${ }^{4}$,

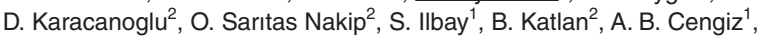
Ö. Basaran ${ }^{3}$, B. C. Cura Yayla ${ }^{4}$, J. Karakaya ${ }^{5}$, Y. Bilginer ${ }^{3}$, B. Bayrakci², M. Ceyhan ${ }^{1}$, S. Özen ${ }^{3}{ }^{1}$ Hacettepe University School of Medicine, Department of Pediatric Infectious Diseases, Ankara, Turkey; ${ }^{2}$ Hacettepe University School of Medicine, Department of Pediatrics, Division of Pediatric Intensive Care Unit, Ankara, Turkey: ${ }^{3}$ Hacettepe University School of Medicine, Department of Pediatrics, Division of Pediatric Rheumatology, Ankara, Turkey; ${ }^{4}$ University of Health Science Ankara Training and Research Hospital, Department of Pediatric Infectious Diseases, Ankara, Turkey; ${ }^{5}$ Hacettepe University School of Medicine, Department of Bioistatistics, Ankara, Turkey

Background: The severity of COVID-19 symptoms can range from mild to severe. Severe COVID-19 cases with excessive hyperinflammation have many overlap features with multisystem inflammatory syndrome in children (MIS-C).

Objectives: We aimed to describe the typical clinical and laboratory features and treatment of children diagnosed with MIS-C and to understand the differences as compared to severe/critical pediatric cases with COVID-19 in an eastern Mediterranean country.

Methods: Children (aged $<18$ years) who diagnosed with MIS-C and severe/ critical pediatric cases with COVID-19, were admitted to hospital between 26 March and 3 November 2020 were enrolled in the study.

Results: A total of 52 patients, 22 patients diagnosed with COVID-19 with severe/critical disease course and 30 patients diagnosed with MIS-C. Although severe COVID-19 cases and cases with MIS-C share many clinical and laboratory features, MIS-C cases had longer fever duration and higher rate of the existence of rash, conjunctival injection, peripheral edema, abdominal pain, altered mental status, and myalgia than in severe cases ( $p<0.001$ for each). Of all, $53.3 \%$ of MIS-C cases had the evidence of myocardial involvement as compared to severe cases (27.2\%). Additionally, C-reactive protein (CRP) and white blood cell (WBC) are the independent predictors for the diagnosis of MIS-C, particularly in the existence of conjunctival injection and rash. Corticosteroids, intravenous immunoglobulin (IVIG), and biologic immunomodulatory treatments were mainly used in MIS-C cases rather than cases with severe disease course. There were only 3 deaths among 52 patients, one of whom had Burkitt lymphoma and the two cases with severe COVID-19 of late referral.
Conclusion: Differences between clinical presentations, acute phase responses, organ involvements, and management strategies indicate that MIS-C might be a distinct immunopathogenic disease as compared to pediatric COVID-19. Conjunctival injection and higher CRP and low WBC count seem good diagnostic parameters for MIS-C cases.

Disclosure of Interests: None declared

DOI: 10.1136/annrheumdis-2021-eular.3572

\section{POS1250 VITAMIN D DEFICIENCY IS MAINLY ASSOCIATED WITH SEVERE LUNG INVOLVEMENT, LONGER DISEASE DURATION AND RISK OF DEATH IN ELDERLY COVID- 19 PATIENTS}

A. Sulli ${ }^{1}$, E. Gotelli ${ }^{1}$, A. Casabella ${ }^{1}$, M. Grosso ${ }^{2}$, C. Schenone ${ }^{1}$, C. Pizzorni ${ }^{1}$, S. Paolino' ${ }^{1}$ E. Alessandri ${ }^{1}$, V. Smith ${ }^{3}$, M. Cutolo' ${ }^{1}{ }^{1}$ Laboratory of Experimental Rheumatology and Academic Division of Clinical Rheumatology, Department of Internal Medicine, University of Genova, IRCCS San Martino Polyclinic Hospital, Genova, Italy; ${ }^{2}$ Pneumology Unit, IRCCS San Martino Polyclinic Hospital, Genova, Italy; ${ }^{3}$ Department of Rheumatology, Ghent University Hospital, Department of Internal Medicine, VIB Inflammation Research Centre Ghent University, Ghent, Belgium

Background: Vitamin D regulates the innate and adaptive immune system responses and low vitamin $\mathrm{D}$ levels have been associated with the increased risk of respiratory tract infections (1). Vitamin $D$ deficiency has been recently reported to interfere with the prognosis of COVID-19 $(2,3)$

Objectives: The aim of this study was to correlate the $25 \mathrm{OH}$-vitamin D serum levels with lung involvement and disease severity, in a cohort of elderly patients hospitalized for SARS-CoV-2 infection.

Methods: Sixty-five COVID-19 patients (mean age $76 \pm 13$ years) and sixty-five sex- and age-matched control subjects (CNT) were included in the study. Respiratory parameters $\left(\mathrm{PaO}_{2}, \mathrm{SO}_{2}, \mathrm{PaCO}, \mathrm{PaO}_{2} / \mathrm{FiO}_{2}\right)$, clinical and laboratory parameters (including $25 \mathrm{OH}$-vitamin D, D-dimer, C-reactive protein) and type of radiological pulmonary involvement were collected at hospital admission. Statistical analysis was performed by non-parametric tests.

Results: Vitamin D sufficiency ( $>30 \mathrm{ng} / \mathrm{ml}$ ), insufficiency (between 20 and $30 \mathrm{ng} /$ $\mathrm{ml})$, deficiency (between 10 and $20 \mathrm{ng} / \mathrm{ml})$ and severe deficiency $(<10 \mathrm{ng} / \mathrm{ml})$ were observed respectively in $11,11,21$ and $57 \%$ of COVID-19 patients. Vitamin D serum levels were found significantly lower in COVID-19 patients than in CNT (median 8 vs $16 \mathrm{ng} / \mathrm{ml}, \mathrm{p}=0.001$ ). A statistically significant positive correlation was observed between vitamin $D$ serum levels and $\mathrm{SO}_{2}(p=0.05), \mathrm{PaO}_{2}(p=0.03)$, $\mathrm{PaO}_{2} / \mathrm{FiO}_{2}(\mathrm{p}=0.02)$. A statistically significant negative correlation was found between vitamin $D$ serum levels and severity of radiologic pulmonary involvement: vitamin D was significantly lower in COVID-19 patients with either diffuse/ severe interstitial lung involvement $(p=0.05)$ or multiple lung consolidations $(p=0.0001)$ than in those with mild radiological lung involvement. Significantly lower vitamin D serum levels were found in COVID-19 patients who died during hospitalization, compared to those who survived (median 3 vs $8 \mathrm{ng} / \mathrm{ml}, \mathrm{p}=0.05$ ) Finally, a statistically significant negative correlation was found between vitamin D serum levels and D-dimer $(p=0.04)$, C-reactive protein $(p=0.04)$ and disease duration $(p=0.05)$

Conclusion: This study confirms that severe vitamin D deficiency is associated with more severe lung involvement, longer disease duration and risk of death in elderly COVID-19 patients.

\section{REFERENCES:}

[1] Cutolo M, et al. RMD Open. 2020; 6(3):e001454.

[2] Bilezikian JP, et al. Eur J Endocrinol. 2020; 183(5):R133-R147.

[3] Weir EK, et al. Clin Med (Lond). 2020; 20:e107-e108.

Disclosure of Interests: None declared

DOI: 10.1136/annrheumdis-2021-eular.3576

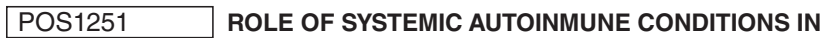 HOSPITAL ADMISSIONS RELATED TO COVID-19}

I. Perez-Sancristobal $^{1}$, L. Lopez Pedraza ${ }^{2}$, M. P. Álvarez Hernandez ${ }^{1}$, J. I. Colomer ${ }^{3}$, A. Madrid García ${ }^{2}$, B. Fernandez ${ }^{1}$, C. Martinez Prada ${ }^{1}$, L. Rodriguez Rodriguez ${ }^{2}$, A. Mucientes ${ }^{2}$, L. León ${ }^{2}$, L. Abasolo ${ }^{2} .{ }^{1}$ Hospital Clínico San Carlos, Reumathology, Madrid, Spain; ${ }^{2}$ Instituto de Investigación Sanitaria del Hospital Clínico San Carlos, Reumathology, Madrid, Spain; ${ }^{3}$ Fundación para la Investigación Biomedica, Reumathology, Madrid, Spain

Background: The COVID-19 pandemic continues worldwide and has had a strong impact on public health, quality of life and economy of the general population. To date, the number of infections and deaths are still increasing. From the beginning of the pandemic, efforts were intensified to identify risk factors for development of the severe form of COVID-19. In this sense underlying medical comorbidities have been shown to have a worse prognosis in these patients. 
Objectives: In patients with rheumatic and musculoskeletal diseases (RMDs) and infected with Covid - 19, we aim to investigate the role of systemic autoimmune conditions compared to other type of RMDs in severity of COVID-19 in terms of hospital admissions.

Methods: An observational longitudinal study was conducted during the epidemic peak in Madrid ( $1^{\text {st }}$ Mar to $20^{\text {th }}$ May2020). All patients attended at the rheumatology outpatient clinic of a tertiary hospital with a diagnosis of RMDs and Covid-19 infection were included (according to a medical diagnosis or confirmed with a positive SARS-CoV-2 PCR diagnostic test). All patients were included since the time of COVID-19 diagnosis. Main variable: hospital admission related to Covid -19 infection. Independent variable: type of RMD including: autoimmune (systemic autoimmune conditions and inflammatory joint disease (IJD)) and nonautoimmune (mechanical diseases, and inflammatory diseases (microcrystalline arthritis and tendonitis)). Covariates: sociodemographic, clinical and therapy used. Statistical analysis: description of the sociodemographic, clinical and treatment characteristics of the patients. A multivariate logistic regression adjusted by age, sex and comorbidities was used to evaluate the risk of the different types of RMDs in hospital admissions related to Covid-19. The results were expressed as OR with its corresponding confidence interval $(95 \% \mathrm{Cl})$.

Results: 406 patients were included with RMDs and Covid- 19 infection. 69.21\% were women with a mean age at diagnosis of $60 \pm 15.26$ years. The evolution time from the diagnosis of RMD was $8 \pm 8.38$ years. $26 \%$ had comorbidity at baseline. $25 \%$ were chronically on corticoids prior to the infection. Of the 406 patients, 244 (60.09\%) had non-autoimmune RMD (157 mechanic, 87 inflammatory) and 162 (39.9\%) (106 (65.43\%) IJD, 56 (34.56\%) systemic autoimmune condition) had autoimmune RMD. $36 \%$ of all patients were admitted (31\% from not autoimmune RMDs and 43\% from autoimmune RMD ( $p=0,013)$. The risk of hospital admission in autoimmune RMD compared to non-autoimmune RMD was higher (OR: 1.68; [1.11-2.54], $p=0.013$ ), being the risk of systemic autoimmune condition compared to both IJD and non-autoimmune RMD higher (OR IJD: 0.41 [0.21-0.51], $p=0.01$; OR non-autoimmune: 0.33; [0.18-0.61]; $p=0.000$ ). After adjusting by confounders, autoimmune RMD had higher risk of hospital admissions compared to the rest (OR: $1.75 ;[1.04-2.95] ; p=0.03)$, and specifically systemic autoimmune condition had higher risk compared to IJD (OR of IJD 0.33; [0.14-.076]; $p=0.009$ ) and compared to non-autoimmune (OR non autoimmune 0.28; [0.13-0.59], $p=0.001$ ). Advanced age (OR: 1.10; [1.07-1.12], $p<0.001$ ), male (OR 0.58; [0.33-1.02], $\mathrm{p}=0.06$ ), and more number of comorbidities (OR 1.39; $[1.02-1.90] p=0.03$ ) also increased the risk of hospitalization related to COVID-19.

Conclusion: One third of the RMD patients infected with COVID-19 required hospital admission. This study shows that patients with autoimmune and specifically with systemic autoimmune conditions have a higher risk of hospitalization related to COVID-19. We also show that advanced age, male sex and a higher number of comorbidities can contribute to worsen the prognosis of the COVID-19 disease.

\section{REFERENCES}

[1] Jorge A, et al. Temporal trends in severe COVID-19 outcomes in patients with rheumatic disease: a cohort study. Lancet Rheumatol. Ahead of print 2021.

[2] Hyrich KL, Machado PM. Rheumatic disease and COVID-19: epidemiology and outcomes. Nature Reviews Rheumatology 2020; 17: 71-72.

[3] Bonfá E, et al. How COVID-19 is changing rheumatology clinical practice. Nat Rev Rheumatol 2021; 17: 11-15.

Disclosure of Interests: None declared

DOI: 10.1136/annrheumdis-2021-eular.3676

\section{POS1252 COVID-19 IN PATIENTS WITH RHEUMATIC DISEASES ON CHRONIC USE OF HYDROXYCHLOROQUINE IN A LARGE BRAZILIAN COHORT - A 24-WEEK PROSPECTIVE STUDY}

G. Salviato Pileggi ${ }^{1}$, G. Ferreira ${ }^{2}$, A. P. Gomides ${ }^{3}$, E. Reis Neto ${ }^{4}$, M. Abreu ${ }^{5}$, C. Albuquerque ${ }^{3}$, N. Araújo $^{6}$, A. B. Bacchiega ${ }^{1}$, D. Bianchi ${ }^{7}$, B. Bica ${ }^{8}$, E. Bonfa $^{9}$, E. Borba ${ }^{9}$, D. Brito ${ }^{10}$, A. Duarte ${ }^{11}$, M. Peixoto Gu e Silva de Souza ${ }^{12}$, K. Wagner Poti Gomes ${ }^{13}$, A. Maria Kakehasi ${ }^{2}$, R. Cavalheiro Do Espírito Santo ${ }^{14}$, P. Realle ${ }^{15}$, E. Klumb ${ }^{16}$, C. C. Lanna ${ }^{2}$, C. Marques ${ }^{11}$, O. Monticielo ${ }^{14}$, L. Mota ${ }^{3}$, G. Munhoz ${ }^{17}$, E. Paiva ${ }^{18}$, H. Pereira ${ }^{19}$, J. R. Provenza ${ }^{20}$, S. Ribeiro ${ }^{19}$, L. Rocha $\mathrm{Jr}^{21}$, C. Sampaio ${ }^{22}, \mathrm{~V}$. Sampaio ${ }^{23}$, E. Sato ${ }^{4}$, T. Laroca Skare ${ }^{24}, \mathrm{~V}$. De Souza ${ }^{15}$, V. Valim ${ }^{25}$, M. Lacerda ${ }^{23}$, R. Xavier ${ }^{14}$, M. Pinheiro ${ }^{26}$. ${ }^{1}$ Faculdade de Medicina de Barretos (FACISB), Education \& Research Institute Cancer Hospital Barretos, Barretos-SP, Brazil, Rheumatology, Barretos-SP, Brazil; ${ }^{2}$ Hospital das Clínicas, Universidade Federal de Minas Gerais (UFMG), Belo Horizonte-MG, Brazil, Rheumatology, Belo Horizonte-MG, Brazil; ${ }^{3}$ Hospital Universitário de Brasilia da Universidade de Brasilia, EBSERH (HUB-UnB), Brasilia-DF, Brazil, Rheumatology, Brasilia-DF, Brazil; ${ }^{4}$ Universidade Federal de São Paulo (UNIFESP/EPM), Rheumatology, São Paulo-SP, Brazil; ${ }^{5}$ Universidade Federal do Rio de Janeiro (UFRJ), Rheumatology, Rio de Janeiro-RJ, Brazil;
${ }^{6}$ Hospital do Servidor Público Estadual, Instituto de Assistência Médica ao Servidor Público Estadual (IAMSPE), São Paulo-SP, Brazil, Rheumatology, São Paulo-SP, Brazil; ${ }^{7}$ Santa Casa de Misericórdia do Rio de Janeiro (HGSCMRJ), Rio de Janeiro-RJ, Brazil, Rheumatology, Rio de Janeiro-RJ, Brazil; ${ }^{8}$ Hospital Universitário Clementino Fraga Filho, Universidade Federal do Rio de Janeiro (UFRJ), Rio de Janeiro-RJ, Brazil, Rheumatology, Rio de Janeiro-RJ, Brazil; ${ }^{9}$ Hospital das Clínicas HCFMUSP, Faculdade de Medicina, Universidade de São Paulo, São Paulo-SP, Brazil, Rheumatology, São Paulo-SP, Brazil; ${ }^{10}$ Hospital Universitário Lauro Wanderley, Universidade Federal da Paraíba (UFPB), João Pessoa-PB, Brazil, Rheumatology, João Pessoa-PB, Brazil; ${ }^{11}$ Hospital das Clínicas da Universidade Federal de Pernambuco (UFPE), Recife-PE, Brazil, Rheumatology, Recife-PE, Brazil; ${ }^{12}$ Santa Casa de Misericórdia de Belo Horizonte, Belo Horizonte-MG, Brazil, Rheumatology, Belo Horizonte-MG, Brazil; ${ }^{13}$ Hospital Geral de Fortaleza (HGF), Fortaleza-CE, Brazil, Rheumatology, Fortaleza-CE, Brazil; ${ }^{14}$ Hospital de Clínicas de Porto Alegre, Universidade Federal do Rio Grande do Sul (UFRGS), Porto Alegre-RS, Brazil, Rheumatology, Porto Alegre-RS, Brazil; ${ }^{15}$ Hospital Universitário da Universidade Federal de Juiz de Fora (UFJF), Juiz de Fora-MG, Brazil, Rheumatology, Juiz de Fora-MG, Brazil; ${ }^{16}$ Hospital Universitário Pedro Ernesto, Universidade do Estado do Rio de Janeiro (UERJ), Rio de Janeiro-RJ, Brazil, Rheumatology, Rio de Janeiro-RJ, Brazil; ${ }^{17}$ Irmandade da Santa Casa de Misericórdia de São Paulo (ISCMSP), São Paulo-SP, Brazil, Rheumatology, São Paulo-SP, Brazil; ${ }^{18}$ Universidade Federal do Paraná (UFPR), Curitiba-PR Brazil, Rheumatology, Curitiba-PR, Brazil; ${ }^{19}$ Hospital Universitário Getúlio Vargas Universidade Federal do Amazonas, Manaus-AM, Brazil, Rheumatology Manaus-AM, Brazil; ${ }^{20}$ Pontifícia Universidade Católica de Campinas (PUCCAMP), Campinas-SP, Brazil, Rheumatology, Campinas-SP, Brazil; ${ }^{21}$ Instituto de Medicina Integral Professor Fernando Figueira (IMIP/ PE), Recife-PE, Brazil, Rheumatology, Recife-PE, Brazil; ${ }^{22}$ Instituto de Ensino e Pesquisa no Sírio Libanês, São Paulo-SP, Brazil, RedCap, São Paulo-SP, Brazil; ${ }^{23}$ Instituto Leônidas \& Maria Deane, Fiocruz, Manaus-AM, Brazil, Epidemiology, Manaus-AM, Brazil; ${ }^{24}$ Hospital Universitário Evangélico Mackenzie (HUEM), Curitiba-PR, Brazil, Rheumatology, Curitiba-PR, Brazil; ${ }^{25}$ Hospital Universitário Cassiano Antonio de Moraes, Universidade Federal do Espírito Santo, Vitória-ES, Brazil, Rheumatology, Vitória-ES, Brazil; ${ }^{26}$ Universidade Federal de São Paulo (UNIFESPIEPM), Rheumatology, São Paulo, Brazil

Background: The role of chronic use of hydroxychloroquine (HCQ) in rheumatic disease (RD) patients during the SARS-CoV-2 pandemic is still subject of discussion.

Objectives: To compare the occurrence of COVID-19 and its outcomes between $\mathrm{RD}$ patients on HCQ use with individuals from the same household not taking the drug during community viral transmission in an observational prospective multicenter study in Brazil.

Methods: Participants were enrolled and monitored through 24-week (From March $29^{\text {th }}$ to Sep $30^{\text {th }}, 2020$ ) regularly scheduled phone calls performed by trained medical professionals. Epidemiological and demographic data, as well as $\mathrm{RD}$ disease activity status and current treatment data, specific information about COVID-19, hospitalization, need for intensive care, and death was recorded in both groups and stored in the Research Electronic Data Capture (REDCap) database. COVID-19 was defined according to the Brazilian Ministry of Health (BMH) criteria. The statistical analysis was performed using IBM-SPSS v.20.0 software. Group comparisons were made using the Man-Whitney, Chi-Square and Fisher Exact Test, as well as multivariate regression models adjusted to confounders. Survival curves were performed using Kaplan-Meier analysis.

Results: A total of 10,427 participants mean age (SD) of 44.04 (14.98) years were enrolled, including 6004 (57.6\%) rheumatic disease patients, of whom $70.8 \%$ had systemic lupus erythematosus (SLE), 6.7\% rheumatoid arthritis (RA), 4\% primary Sjögren's syndrome (pSS), 1.8\% mixed connective tissue disease (DMTC) $1 \%$ systemic sclerosis (SSc) and others (15.9), including overlap syndromes In total, $1,132(10.8 \%)$ participants fulfilled criteria for COVID-19, being $6.7 \%$ RD patients and $4.1 \%$ controls $(p=0.002)$. A recent influenza vaccination had a protective role $(p<0.001)$. Moderate and severe COVID-19 included the need for hospitalization, intensive care, mechanical ventilation or death. Infection severity was not different between groups $(p=0.391)$ (Table 1). After adjustments for multiple confounders, the main risk factors significantly associated with COVID-19 were higher education level $(\mathrm{OR}=1.2995 \% \mathrm{Cl} 1.05-1.59)$, being healthcare professionals (OR=1.91; $95 \% \mathrm{Cl} 1.45-2.53)$, presence of two comorbidities $(\mathrm{OR}=1.31$ $95 \% \mathrm{Cl} 1.01-1.66)$ and three or more comorbidities associated (OR=1.69; $95 \% \mathrm{Cl}$ $1.23-2.32)$. Interestingly, age $>=65$ years $(\mathrm{OR}=0.20 ; 95 \% \mathrm{Cl} 0.11-0.34)$ was negatively associated. Regarding RD, the risk factors associated with COVID-19 diagnosys were SLE $(\mathrm{OR}=2.37 ; 95 \% \mathrm{Cl} 1.92-293)$, SSc $(\mathrm{OR}=2.25 ; 95 \% \mathrm{Cl} 1.05$ $4.83)$ and rituximab use $(\mathrm{OR}=1.92 ; 95 \% \mathrm{Cl} 1.13-3.26)$. In addition, age $>=65$ years $(\mathrm{OR}=5.47 ; 95 \% \mathrm{Cl} 1.7-19.4)$ and heart disease $(\mathrm{OR}=2.60 ; 95 \% \mathrm{Cl} 1.06-6.38)$ were associated with hospitalization. Seven female RD patients died, six with SLE and one with pSS, and the presence of two or more comorbidities were associated with higher mortality rate. 